\title{
Effective field theory calculation of second post-Newtonian binary dynamics
}

\author{
James B. Gilmore* and Andreas Ros: \\ Department of Physics, Yale University, New Haven, Connecticut 06520, USA
}

(Dated: October 23, 2018)

\begin{abstract}
We use the effective field theory for gravitational bound states, proposed by Goldberger and Rothstein, to compute the interaction Lagrangian of a binary system at the second post-Newtonian order. Throughout the calculation, we use a metric parametrization based on a temporal KaluzaKlein decomposition and test the claim by Kol and Smolkin that this parametrization provides important calculational advantages. We demonstrate how to use the effective field theory method efficiently in precision calculations, and we reproduce known results for the second post-Newtonian order equations of motion in harmonic gauge in a straightforward manner.
\end{abstract}

\section{INTRODUCTION}

In the last two decades, significant progress has been made towards the detection of gravitational waves (GWs) via laser interferometry. Currently, the ground-based experiments LIGO [1], VIRGO [2], GEO 3], and TAMA [4] are actively searching for GWs [5]. Moreover, the proposed LISA experiment [6], due to be the first space-based GW detector, will search for GWs in a complementary frequency band to the ground-based experiments and is expected to achieve high event rates at an unprecedented signal-to-noise ratio [7].

A particularly interesting source of GWs, which is expected to be detected, is the compact binary system undergoing coalescence, with neutron star (NS) and/or black hole (BH) constituents. Current experiments have yet to detect the binary inspiral signal. However, Advanced LIGO [8], an upgrade of LIGO scheduled to come online in 2014, may allow for routine detection of such events. This is due to a $\sim 10$-fold increase in sensitivity over LIGO, which will in turn result in an increase of the accessible event rate by a factor $\sim 1000$. Current estimates for the number of expected NS/NS, BH/BH, and BH/NS events in Advanced LIGO are roughly $10-100,1-500$, and $1-30$ per year, respectively [9, 10].

All three stages of the binary coalescence, inspiral, merger, and ringdown, are potentially detectable. The inspiral phase, where the characteristic orbital velocity is $v^{2} \ll 1$ (in units where $c=1$ ), can be computed analytically using an expansion in $v^{2} \sim G m / r$. The merger is computed numerically [11], and there has been significant recent progress in this area [12]. The ringdown can be treated analytically using quasinormal modes [13].

The perturbative calculation of the inspiral phase has been performed with a variety of methods [14, 15]. Because of the phase evolution of the inspiral signal and the ability to measure the total orbital phase to $\sim 10^{-3}$ over the LIGO bandwidth [16], these perturbation expansions must be calculated to high order. If we consider a circular orbit in the adiabatic approximation, the signal phase $\Phi(\omega)$ is related to the orbital energy $E(\omega)$ and the radiated power $P(\omega)$ through the relation $d^{2} \Phi / d \omega^{2} \sim(d E / d \omega) / P$. An accuracy of $\sim 10^{-3}$ in the cumulative orbital phase, over the LIGO bandwidth, can be achieved if the perturbation expansion is calculated to $\mathcal{O}\left(v^{6}\right)$ beyond Newtonian dynamics i.e., at third post-Newtonian order $(3 \mathrm{PN})[9,17]$. This implies that we need to know $E(\omega)$ and $P(\omega)$ to at least $3 \mathrm{PN}$. Since the conservative dynamics, described in our formalism by a Lagrangian, gives $E(\omega)$, it also must be known to $3 \mathrm{PN}$. There is also a need to compute the perturbative expansion to high order, to allow numerical studies of the binary inspiral to be compared with the analytic PN expansion [18].

Recently, Goldberger and Rothstein introduced an effective field theory (EFT) for nonrelativistic gravitational systems, known as NRGR [19, 20] (see 21] for a pedagogical introduction). EFTs are particularly well suited to problems with multiple physical scales, and the binary inspiral which we consider here, is one such problem. The hierarchy during inspiral takes the form $r_{c} \ll r \ll \lambda$, where $r_{c}$ is the radius of the compact objects, $r$ is the

*Electronic address: james.gilmore@yale.edu

$\dagger^{\dagger}$ Electronic address: andreas.ross@yale.edu 
orbital separation, and $\lambda$ is the wavelength of the emitted GWs. One can use this hierarchy to set up a tower of EFTs which systematically account for effects at each scale. This approach disentangles the physical effects of different scales, resulting in more tractable calculations. With the definite power-counting scheme established by Goldberger and Rothstein [19], the EFT treatment yields a completely systematic description of the binary inspiral problem. In particular, divergences which arise by using point particle sources to represent the compact objects are well understood in a field theory setting, and the effects due to the spatial extent of the compact objects can be systematically parameterized by subleading operators.

State-of-the-art calculations using traditional methods have yielded the conservative equations of motion and the orbital energy of a binary with spinless constituents to 3PN order [22, 23, 24, 25]. The 2PN dynamics of a three-body system have been obtained in [26], but the four-body dynamics at 2PN are not known in closed form, see, for example, Appendix D of [27]. Furthermore, the power emitted by a binary with spinless objects through GWs is known at the 3.5PN level [28] or $\mathcal{O}\left(v^{7}\right)$ beyond the leading quadrupole formula. While the EFT approach lags behind the traditional post-Newtonian methods in terms of high precision calculations of GW observables for binaries without spin, it has led to a number of interesting results. Spin was incorporated into the EFT framework and the next-to-leading order (NLO) spin-spin potential, which enters at 3PN, has been calculated for the first time [29]. Dissipative effects such as absorption by $\mathrm{BH}$ horizons have also been considered [30]. In [31], the thermodynamics of compactified black holes were studied, and the Einstein-Infeld-Hoffman (EIH) Lagrangian and the quadrupole formula were derived in arbitrary dimensions in [32]. The EFT formalism was extended to the case of extreme mass-ratio binaries where the leading order (LO) self-force equation was derived [33]. Recently, it has been used in calculations which include interactions beyond Einstein's general relativity [34, 35].

Within the EFT method, Kol and Smolkin (KS) suggested that a temporal Kaluza-Klein parametrization of the metric [36] improved the calculational efficiency of NRGR. The KS parametrization has been shown to reduce the complexity required to calculate the EIH Lagrangian [37]. Similar simplifications were observed in the computation of thermodynamic properties of compactified black holes [36], and in the calculation of the NLO order spin-spin potential [38].

In this paper, we report the NRGR calculation of the 2PN interaction Lagrangian for a binary system with nonspinning compact objects. Existing EFT calculations of binary observables have been obtained at NLO for potential interactions and at $\mathrm{LO}$ for the radiated power. This work presents the first next-to-next-to-leading order computation of a GW observable with the EFT method. Since the complexity of our calculation is more involved, we clearly want to find an optimum method to perform high precision calculations within NRGR. Previously, it was not clear how useful the KS parametrization would be as a computational tool beyond NLO. We address this issue in detail, and we show how the KS parametrization simplifies our $2 \mathrm{PN}$ calculations. The methods of EFT are used to systematically determine all relevant Feynman diagrams which contribute at 2PN. In their evaluation, we encounter Feynman integrals corresponding to one-loop and two-loop integrals, which are computed using standard techniques. Our work demonstrates how the EFT method to can be used to efficiently compute conservative dynamics at high precision in the PN expansion.

\section{SETUP}

Here, we outline the ingredients of our calculation of the $2 \mathrm{PN}$ potential and discuss the simplifications we will employ. In this section, we do not repeat in entirety the setup of the EFT description of the binary inspiral problem, but refer the reader to [19], 20], and [21]. Since we deal with conservative dynamics, we only need potential modes, and can simply set the radiation modes to zero.

\section{A. Two-body action}

The purely gravitational action is the usual Einstein-Hilbert action

$$
S_{E H}=-2 m_{P l}^{2} \int d^{4} x \sqrt{-g} R,
$$


where our conventions are $m_{P l}^{2}=1 / 32 \pi G, \eta_{\mu \nu}=\operatorname{diag}[1,-1,-1,-1], R_{\nu \alpha \beta}^{\mu}=\partial_{\alpha} \Gamma_{\nu \beta}^{\mu}-\partial_{\beta} \Gamma_{\nu \alpha}^{\mu}+\Gamma_{\lambda \alpha}^{\mu} \Gamma_{\nu \beta}^{\lambda}-\Gamma_{\lambda \beta}^{\mu} \Gamma_{\nu \alpha}^{\lambda}$, and $R_{\mu \nu}=R_{\mu \alpha \nu}^{\alpha}$. In addition, we must also fix the gauge. Our choice is harmonic gauge

$$
S_{G F}=m_{P l}^{2} \int d^{4} x \sqrt{-g} \Gamma^{\mu} \Gamma^{\nu} g_{\mu \nu}
$$

where $\Gamma^{\mu}=\Gamma_{\alpha \beta}^{\mu} g^{\alpha \beta}$. It differs from the linearized harmonic gauge condition used in [19] and yields different expressions at the $2 \mathrm{PN}$ level. The advantage of using harmonic gauge is that we can compare intermediate gauge-dependent results, such as the equations of motion, with the existing literature [14].

For the gravitational coupling to two massive compact objects, the worldline action for our binary system is given by

$$
S_{p p}=-\sum_{N=1}^{2} m_{N} \int d \tau_{N}+\cdots=-\sum_{N=1}^{2} m_{N} \int d t \sqrt{g_{\mu \nu}\left(t, \mathbf{x}_{N}\right) \frac{d x_{N}^{\mu}}{d t} \frac{d x_{N}^{\nu}}{d t}}+\cdots
$$

Here, proper time is given by $d \tau^{2}=g_{\mu \nu} d x^{\mu} d x^{\nu}$ and it is convenient to use coordinate time $t$ to parameterize the worldline. The dots denote subleading operators encoding finite size effects. We will ignore finitesize effects since they first enter at 5PN [19].

\section{B. Kol-Smolkin variables}

The expansion of the metric around flat Minkowski space in the weak field limit is commonly parameterized in a Lorentz covariant form such as

$$
g_{\mu \nu}=\eta_{\mu \nu}+h_{\mu \nu} / m_{P l}
$$

where $h_{\mu \nu}$ is the excitation around Minkowski space. The expansion of Eq. (4) was used in the original setup by Goldberger and Rothstein [19]. Instead, we will use an alternative parametrization based on a temporal Kaluza-Klein decomposition, as suggested by KS [36]. In terms of the KS variables, the metric reads

$$
g_{\mu \nu}=\left(\begin{array}{cc}
e^{2 \phi / m_{P l}} & -e^{2 \phi / m_{P l}} A_{j} / m_{P l} \\
-e^{2 \phi / m_{P l}} A_{i} / m_{P l} & -e^{-2 \phi / m_{P l}} \gamma_{i j}+e^{2 \phi / m_{P l}} A_{i} A_{j} / m_{P l}^{2}
\end{array}\right)
$$

where $\gamma_{i j}=\left(\delta_{i j}+\sigma_{i j} / m_{P l}\right)$. In four dimensions, the metric excitations are now described in terms of a scalar field $\phi$, a three-vector field $A_{i}$, and a $3 \times 3$ symmetric tensor field $\sigma_{i j}$, all of which have been normalized to have mass dimension 1. In the ground state, $\langle\phi\rangle=\left\langle A_{i}\right\rangle=\left\langle\sigma_{i j}\right\rangle=0$, and the metric reduces to Minkowski space-time. In terms of the $\mathrm{KS}$ variables, the $\mathrm{EH}$ action has the simple form

$$
S_{E H}=-2 m_{P l}^{2} \int d^{4} x \sqrt{\gamma}\left(-R[\gamma]-\frac{1}{4} e^{4 \phi} F_{i j} F^{i j}+2\left(\partial^{i} \phi\right)\left(\partial_{i} \phi\right)+\ldots\right),
$$

where $F_{i j}=\partial_{i} A_{j}-\partial_{j} A_{i}$ and the dots denote terms with time derivatives. In Eq. (6) all spatial indices are lowered or raised with $\gamma_{i j}$ or $\gamma^{i j}$, respectively. Some of the terms involving time derivatives are needed for our calculation along with the expansion of the gauge fixing action of Eq. (2), and their contributions enter the Feynman rules given in Sec. III

Given the gravitational action Eqs. (1) and (2), the worldline action from Eq. (3), and the KS metric parametrization from Eq. (5), we can proceed to derive Feynman rules for the gravitational self-couplings and the couplings to the compact object worldlines. The procedure is equivalent to the one in [19], but is now implemented in terms of the KS fields. For the gravitational self-couplings and worldline couplings, there are infinitely many terms in the respective actions. To avoid unnecessary calculation at a given order, a method of systematic computation must be established. 


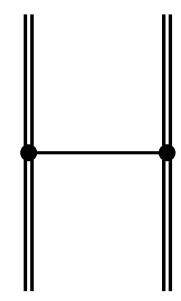

FIG. 1: Order $G$ topology. The single solid line denotes a generic graviton field, either $\phi, A_{i}$, or $\sigma_{i j}$, and double solid lines denote the worldlines of the binary constituents.

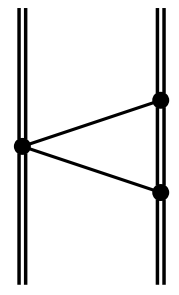

$(a)$

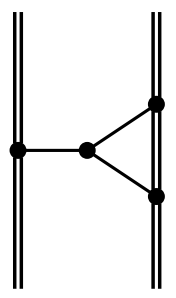

(b)

FIG. 2: Topologies at order $G^{2}$.

\section{Power counting and Feynman diagram topologies}

The power-counting rules for our calculation are the same as those in the original EFT setup developed in [19]. However, since there are no radiation fields in our $2 \mathrm{PN}$ potential calculation, we use a simplified procedure where we power count our Feynman diagrams relative to the LO Newtonian potential. For a bound state, the virial theorem relates the orbital velocity $v$ and Newton's constant $G$ via, $v^{2} \sim G m / r$, and as shown in [19], one can power count all contributions in powers of the orbital velocity $v$. Keeping the virial theorem in mind, we count powers of $G$ and powers of $v^{2}$ separately. For the power counting we do not keep track of factors of mass $m$ and separation $r$ associated to each $G$. These will be generated appropriately in the calculation of the diagrams. In this scheme, the diagram responsible for the Newtonian potential scales as $\mathcal{O}\left(G v^{0}\right)$. For the $2 \mathrm{PN}$ potential calculation, we need to include all diagrams which scale as $\mathcal{O}\left(G v^{4}\right), \mathcal{O}\left(G^{2} v^{2}\right)$, and $\mathcal{O}\left(G^{3} v^{0}\right)$.

To determine the relevant Feynman diagrams at $2 \mathrm{PN}$, the first stage is to generate all relevant diagram topologies. To do this, one first counts in powers of $G$, since all interaction vertices will scale with a power of $G$ (recall $m_{P l} \sim G^{-1 / 2}$ ). Powers of velocity are inserted later, when all relevant diagram topologies have been established. There are two rules to consider when counting a topology in factors of $G$. Firstly, when there are $n$ gravitons attached to a worldline, this component receives a factor of $G^{n / 2}$. Secondly, each $n$-graviton self-interaction vertex receives a factor of $G^{n / 2-1}$. Note that a propagator does not receive any factors. Using these rules any diagram topology can be counted in powers of $G$.

If we now proceed to apply these rules, we can only have a single topology at $\mathcal{O}(G)$, as shown in Fig. 1. All topologies which simply renormalize the mass are omitted, as discussed in [19]. Diagrams which involve a graviton line starting and ending on the same worldline, without any intermediate interaction, fall into this category. At the next order, $\mathcal{O}\left(G^{2}\right)$, we have two topologies, as shown in Fig. 2, Since the worldlines are static sources and do not propagate, the diagrams in Fig. 2 do not involve any loops. Diagrams with gravitational loops yield quantum effects and are therefore ignored, and since only massless fields propagate in NRGR, the expansion in loops corresponds to an expansion in powers of $\hbar$ [3]. At $\mathcal{O}\left(G^{3}\right)$, the total number of possible topologies is nine, as shown in Fig. 3. These diagrams are the relevant topologies for the static component of the $2 \mathrm{PN}$ potential. In Figs. 2 and 3 , we have not drawn the topologies with the two worldlines interchanged, although they will be required for our calculations.

Now that we have established all topologies relevant to the calculation at $2 \mathrm{PN}$, the powers of the orbital velocity $v$ must be counted. There are two possible sources for factors of $v$ in our calculations: (1) fields coupling to the worldlines, where the LO couplings of $\phi, A_{i}$, and $\sigma_{i j}$ are $\mathcal{O}\left(v^{0}\right), \mathcal{O}\left(v^{1}\right)$, and $\mathcal{O}\left(v^{2}\right)$, respectively, and (2) time derivatives, where 


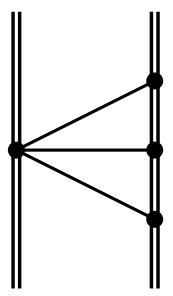

(a)

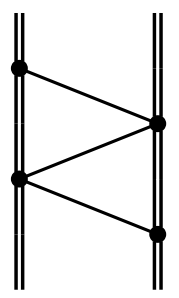

(e)

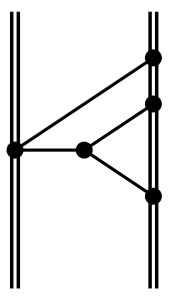

(b)

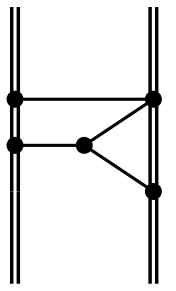

(f)

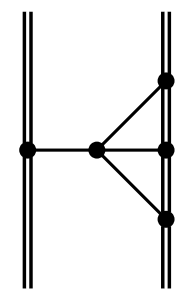

(c)

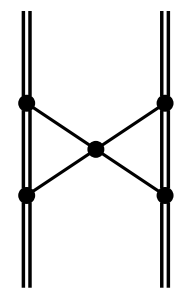

(g)

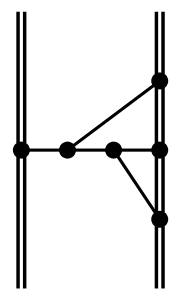

(d)

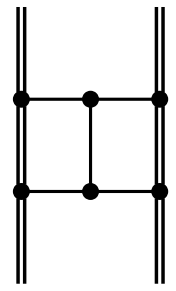

(h)

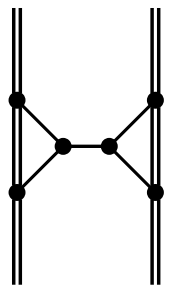

(i)

FIG. 3: Topologies at order $G^{3}$.

$\partial_{0} \sim v / r$ for potential modes. Time derivatives can arise from either purely gravitational interaction vertices or from propagator insertions, where each propagator insertion counts as $\mathcal{O}\left(v^{2}\right)$.

With all relevant topologies and the counting rules for $v$, the final diagrams at $2 \mathrm{PN}$ can now be constructed. Recall that the 2PN diagrams scale as either $\mathcal{O}\left(G v^{4}\right), \mathcal{O}\left(G^{2} v^{2}\right)$, or $\mathcal{O}\left(G^{3} v^{0}\right)$. The diagrams are generated by first populating the topologies with the three gravitational fields $\phi, A_{i}$, and $\sigma_{i j}$ in all possible combinations. We then count the factors of $v$ which result from the gravitational fields coupling to the worldline, and any time derivatives acting on internal vertices. Propagator insertions are also counted where appropriate. The powers of $v$ from the worldline vertices are kept to all orders in the calculations and only expanded to $2 \mathrm{PN}$ in the final result. The diagrams relevant at $2 \mathrm{PN}$ which result from this procedure are shown in Sec. III. Figs. 4, 5, and 6.

\section{Advantages of the Kol-Smolkin variables}

In the KS parametrization of Eq. (5), we have introduced three fields, $\phi, A_{i}$, and $\sigma_{i j}$, instead of the standard $h_{\mu \nu}$ parametrization of Eq. (4). It will be necessary to keep track of these new fields, so one should ask what has been gained from a calculational perspective by using the KS parametrization. This question is answered by considering the static diagrams, i.e. those with no velocity factors. First, consider the topology Fig. $2(b)$ which involves a threegraviton vertex. This was used in [19] to construct the EIH potential in the $h_{\mu \nu}$ variables. When the KS variables are employed, it was shown that this topology does not contribute at 1PN order [37]. We discuss this in more detail since it points us to the source of the advantages of the KS variables.

When working in the $h_{\mu \nu}$ parametrization, one often considers its components $h_{00}, h_{0 i}$, and $h_{i j}$ separately, since their LO coupling to the worldline scale as $\mathcal{O}\left(v^{0}\right), \mathcal{O}\left(v^{1}\right)$, and $\mathcal{O}\left(v^{2}\right)$, respectively. So in practice, in the $h_{\mu \nu}$ and KS parametrizations, one must keep track of the same number of components or fields. Returning to our discussion, if we consider static diagrams, only the $h_{00}$ component can couple to the worldline. Let us consider the static limit of the first topology which arises with gravitational self-couplings, Fig. $2(b)$. With $h_{00}$ coupling to each worldline at LO, without any propagator insertions, the only possible source of powers of $v$ would be the three-graviton vertex. Naively, one would isolate the action component with three powers of $h_{00}$, which yields the $3-h_{00}$ vertex. Interestingly, one finds that the corresponding term in the action is proportional to $h_{00}\left(\partial_{0} h_{00}\right)^{2}$, meaning that the 3 - $h_{00}$ vertex has a power of $v^{2}$ associated with it. So does this mean that the topology in Fig. 2 $2(b)$ does not enter at 1PN in the $h_{\mu \nu}$ parametrization? If this were true, then the EIH calculation of [19] would be contradicted. The reason why the above conclusion is wrong is the presence of mixing of the $h_{\mu \nu}$ components. 
To explain this point, consider the $h_{\mu \nu}$ graviton propagator in harmonic gauge, which is given by $\left\langle T h_{\mu \nu} h_{\alpha \beta}\right\rangle \sim$ $\left(\eta_{\mu \alpha} \eta_{\nu \beta}+\eta_{\mu \beta} \eta_{\nu \alpha}-\eta_{\mu \nu} \eta_{\alpha \beta}\right)$. Examining the tensor structure of the propagator, one can see $h_{00}$ mixes with the trace of $h_{i j}$, since $\left\langle T h_{00} h_{i j}\right\rangle \sim \delta_{i j}$. Thus, a graviton which starts as $h_{00}$ at the worldline coupling can be either $h_{00}$ or $h_{i i}$ at the three-graviton vertex. So instead of the $3-h_{00}$ vertex, one has to use the three-point function $\left\langle T h_{00} h_{00} h_{00}\right\rangle$. This three-point function is obtained by contracting the $3-h_{\mu \nu}$ vertex with three graviton propagators and setting all remaining free indices to 0 . One then finds that there are components of the three-point function $\left\langle T h_{00} h_{00} h_{00}\right\rangle$ which do not involve time derivatives, and so the topology Fig. 2(b) is required at $1 \mathrm{PN}$ in the $h_{\mu \nu}$ parametrization. Clearly, the topology Fig. $2(b)$ only survives at $1 \mathrm{PN}$ in the $h_{\mu \nu}$ parametrization because $h_{00}$ can mix with $h_{i i}$ as it propagates.

The major advantage of the KS parametrization in harmonic gauge is that it removes this kind of mixing, since the two-point functions between the three fields are zero: $\left\langle T \phi A_{i}\right\rangle=\left\langle T \phi \sigma_{j k}\right\rangle=\left\langle T A_{i} \sigma_{j k}\right\rangle=0$. For the topology Fig. $2(b)$ to contribute to the EIH potential in the KS parametrization, all of the fields coupling to the worldlines must be $\phi$ 's, since only they couple at $\mathcal{O}\left(v^{0}\right)$. Knowing that $\phi$ cannot mix with $A_{i}$ or $\sigma_{i j}$, the 3 - $\phi$ vertex must be used. When the gravitational Lagrangian is examined, the only relevant term is proportional to $\phi \dot{\phi}^{2}$, where the two time derivatives in this term cause the $3-\phi$ vertex to be of $\mathcal{O}\left(v^{2}\right)$ in the orbital velocity. Therefore, the diagrams resulting from the topology Fig. 2(b) do not contribute until 2PN in the KS parametrization.

This argument can be extended to the $n$ - $\phi$ vertex $(n>2)$, where the relevant term in the gravitational action is $\sim \exp \left(-4 \phi / m_{P l}\right) \dot{\phi}^{2}$. Any $n-\phi$ vertex is then suppressed by one order in the PN expansion because there are always two time derivatives. Thus, in the KS parametrization, the $\mathcal{O}\left(G^{3}\right)$ topologies Fig. $3(b),(c),(f)$, and $(g)$ will not enter at $2 \mathrm{PN}$. This will reduce the calculational effort considerably. Also, the fact that the topology Fig. $2(b)$ first enters at $2 \mathrm{PN}$, rather than $1 \mathrm{PN}$, means that propagator insertions are not required for this topology at $2 \mathrm{PN}$.

We have established that the KS variables in harmonic gauge provide important calculational advantages over the $h_{\mu \nu}$ parametrization used in [19] at $2 \mathrm{PN}$ order. At $1 \mathrm{PN}$, this advantage was modest, since only one diagram topology was eliminated. At $2 \mathrm{PN}$, we have found that four topologies at $\mathcal{O}\left(G^{3} v^{0}\right)$ were removed, and one did not need propagator insertions at $\mathcal{O}\left(G^{2} v^{2}\right)$ on the topology Fig. 2(b). Clearly as we proceed to higher order in the PN expansion, the advantages of the KS variables become more important.

\section{CALCULATION OF FEYNMAN DIAGRAMS}

In this section, we present the calculation of the Feynman diagrams which are required to determine the $2 \mathrm{PN}$ interaction potential between the binary constituents. Using the Feynman rules presented below, each diagram equals $-i \int d t V$ from which we extract its contribution to the potential $V$. The symmetry factor for each diagram is computed in the usual manner [40], but one needs to account for the nonpropagating classical sources. As discussed in Sec. IIC] there are three classes of diagrams with the following power counting: $\mathcal{O}\left(G^{3} v^{0}\right), \mathcal{O}\left(G^{2} v^{2}\right)$, and $\mathcal{O}\left(G v^{4}\right)$. We discuss how to evaluate each in turn, starting with the simplest at $\mathcal{O}\left(G v^{4}\right)$, and finishing with the more complex diagrams at $\mathcal{O}\left(G^{3} v^{0}\right)$.

When calculating the diagrams, we work with the spatial Fourier transform for the gravitational potential modes. For example, for the $\phi$ field, we will work in terms of $\phi_{\mathbf{k}}(t)$, which is the spatial Fourier transform of the coordinate space field $\phi(t, \mathbf{x})=\int_{\mathbf{k}} e^{i \mathbf{k} \cdot \mathbf{x}} \phi_{\mathbf{k}}(t)$, where $\int_{\mathbf{k}}=\int \frac{d^{3} \mathbf{k}}{(2 \pi)^{3}}$. We also make clear that after expanding the metric in the KS fields $\phi, A_{i}$, and $\sigma_{i j}$, we do not distinguish between upper and lower spatial indices; they are lowered and raised with $\delta_{i j}$ and $\delta^{i j}$, respectively. Lastly, since we are interested in the long range potential only, we drop irrelevant contact terms, such as $V \sim \delta(r)$, wherever they appear in the calculations.

\section{A. Order $G v^{4}$ diagrams}

As shown in Fig. 4, there are six diagrams to be evaluated at $\mathcal{O}\left(G v^{4}\right)$. The contributions from the diagrams $(a)$, $(d)$, and $(f)$ are the easiest of all diagrams to compute at $2 \mathrm{PN}$, since we only need to compute simple one-graviton exchange diagrams.

The Feynman rules for couplings to the worldlines are derived from the point particle action in Eq. (3), which is expanded in the gravitational fields to the required order for a given Feynman rule. The Feynman rules for a $\phi, A_{i}$, 


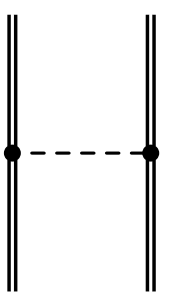

(a)

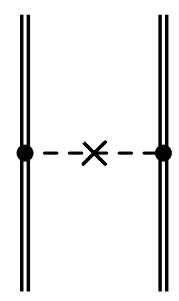

(b)

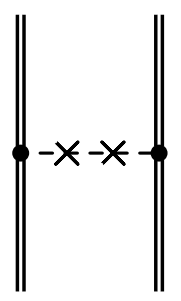

$(c)$

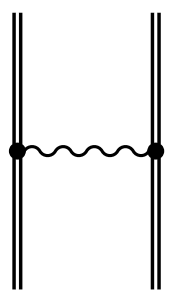

$(d)$

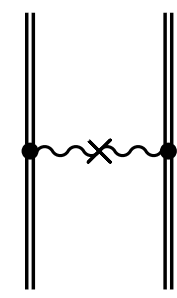

$(e)$

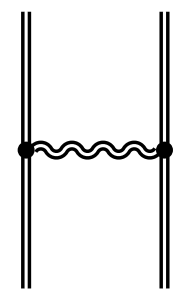

$(f)$

FIG. 4: Order $G v^{4}$ diagrams at $2 \mathrm{PN}$. Here the dashed, wavy, and double wavy lines, represent the $\phi, A_{i}$, and $\sigma_{i j}$ fields, respectively. A cross denotes a propagator insertion.

and $\sigma_{i j}$ coupled to the worldline are

$$
\begin{aligned}
& \sim \sim=-\frac{i m}{m_{P l}} \int d t \int_{\mathbf{k}} e^{i \mathbf{k} \cdot \mathbf{x}} \frac{1+\mathbf{v}^{2}}{\sqrt{1-\mathbf{v}^{2}}} \\
& \sim=\frac{i m}{m_{P l}} \int d t \int_{\mathbf{k}} e^{i \mathbf{k} \cdot \mathbf{x}} \frac{\mathbf{v}_{i}}{\sqrt{1-\mathbf{v}^{2}}} \\
& \sim=\frac{i m}{2 m_{P l}} \int d t \int_{\mathbf{k}} e^{i \mathbf{k} \cdot \mathbf{x}} \frac{\mathbf{v}_{i} \mathbf{v}_{j}}{\sqrt{1-\mathbf{v}^{2}}} .
\end{aligned}
$$

The double solid lines are the worldlines, and the dashed, wavy, and double wavy lines represent the $\phi, A_{i}$, and $\sigma_{i j}$ fields, respectively. Note that these rules are exact in $v$. We will expand in $v$ at the end of the calculation to isolate the required terms at $2 \mathrm{PN}$.

To compute diagrams $(a),(d)$, and $(f)$, we now need the propagators for each gravitational field. From the purely gravitational action, the sum of Eqs. (11) and (2), the propagators can be derived. These potential propagators are nonrelativistic and instantaneous, and for the $\phi, A_{i}$, and $\sigma_{i j}$ gravitational fields we have, respectively,

$$
\begin{aligned}
\left\langle T \phi_{\mathbf{p}}\left(t_{a}\right) \phi_{\mathbf{q}}\left(t_{b}\right)\right\rangle & =-\frac{1}{8}(2 \pi)^{3} \delta^{3}(\mathbf{p}+\mathbf{q}) \frac{i}{\mathbf{p}^{2}} \delta\left(t_{a}-t_{b}\right) \\
\left\langle T A_{\mathbf{p}}^{i}\left(t_{a}\right) A_{\mathbf{q}}^{j}\left(t_{b}\right)\right\rangle & =\frac{1}{2}(2 \pi)^{3} \delta^{3}(\mathbf{p}+\mathbf{q}) \frac{i \delta^{i j}}{\mathbf{p}^{2}} \delta\left(t_{a}-t_{b}\right) \\
\left\langle T \sigma_{\mathbf{p}}^{i j}\left(t_{a}\right) \sigma_{\mathbf{q}}^{k l}\left(t_{b}\right)\right\rangle & =-(2 \pi)^{3} \delta^{3}(\mathbf{p}+\mathbf{q}) \frac{i P^{i j, k l}}{\mathbf{p}^{2}} \delta\left(t_{a}-t_{b}\right),
\end{aligned}
$$

where $P^{i j, k l}=\frac{1}{2}\left(\delta^{i k} \delta^{j l}+\delta^{i l} \delta^{j k}-2 \delta^{i j} \delta^{k l}\right)$.

The three single-graviton exchange diagrams are easily computed with the above propagators and worldline couplings. At $\mathcal{O}\left(G v^{4}\right)$, the only integral in $\mathbf{k}$ which arises is a Fourier transform, which is evaluated using the $d$-dimensional formula

$$
\int \frac{d^{d} \mathbf{k}}{(2 \pi)^{d}} \frac{1}{\left(\mathbf{k}^{2}\right)^{\alpha}} e^{i \mathbf{k} \cdot \mathbf{r}}=\frac{1}{(4 \pi)^{d / 2}} \frac{\Gamma(d / 2-\alpha)}{\Gamma(\alpha)}\left(\frac{\mathbf{r}^{2}}{4}\right)^{\alpha-d / 2}
$$


Doing the Fourier transform, and extracting the 2PN component, gives the following potential contributions:

$$
\begin{aligned}
V^{(a)} & =-\frac{G m_{1} m_{2}}{r}\left(\frac{7}{8} \mathbf{v}_{1}^{4}+\frac{9}{4} \mathbf{v}_{1}^{2} \mathbf{v}_{2}^{2}+\frac{7}{8} \mathbf{v}_{2}^{4}\right) \\
V^{(d)} & =-\frac{G m_{1} m_{2}}{r}\left(-2 \mathbf{v}_{1} \cdot \mathbf{v}_{2}\left(\mathbf{v}_{1}^{2}+\mathbf{v}_{2}^{2}\right)\right) \\
V^{(f)} & =-\frac{G m_{1} m_{2}}{r}\left(2\left(\mathbf{v}_{1} \cdot \mathbf{v}_{2}\right)^{2}-2 \mathbf{v}_{1}^{2} \mathbf{v}_{2}^{2}\right)
\end{aligned}
$$

where the notation we use here and subsequently is $\mathbf{r} \equiv \mathbf{x}_{1}(t)-\mathbf{x}_{2}(t), r \equiv|\mathbf{r}|$, and $\mathbf{n} \equiv \mathbf{r} / r$. The labels 1 and 2 are used for the left and right worldlines, respectively. Although $\mathbf{r}, \mathbf{v}_{1}$, etc., depend on $t$, we have suppressed this dependence above. We will continue to do this when convenient.

Diagrams $(b),(c)$, and $(e)$ all involve propagator insertions. These insertions account for the corrections to the instantaneous nature of the nonrelativistic potential propagators in Eqs. (10) - (12). These are included systematically as a perturbation, where each propagator insertion is suppressed with respect to the propagator by a power of $v^{2}$. The insertions are obtained from the terms in the action which are quadratic in the fields and which involve time derivatives. The required propagator insertions at $2 \mathrm{PN}$ are derived to be

$$
\begin{aligned}
& ----X---=-\frac{1}{8}(2 \pi)^{3} \delta^{3}(\mathbf{p}+\mathbf{q}) \frac{i}{\mathbf{p}^{4}} \partial_{t_{a}} \partial_{t_{b}} \delta\left(t_{a}-t_{b}\right) \\
& --\not--メ--=-\frac{1}{8}(2 \pi)^{3} \delta^{3}(\mathbf{p}+\mathbf{q}) \frac{i}{\mathbf{p}^{6}} \partial_{t_{a}}^{2} \partial_{t_{b}}^{2} \delta\left(t_{a}-t_{b}\right) \\
& \sim \sim \sim=\frac{1}{2}(2 \pi)^{3} \delta^{3}(\mathbf{p}+\mathbf{q}) \frac{i \delta^{i j}}{\mathbf{p}^{4}} \partial_{t_{a}} \partial_{t_{b}} \delta\left(t_{a}-t_{b}\right),
\end{aligned}
$$

where one cross denotes a single propagator insertion and two crosses denote two propagator insertions. Here, we have chosen the simplest symmetric form of the time derivatives; however, other choices are possible. Note that any other choice of time derivatives yields potentials which can be related to potentials computed with the simplest symmetric choice by a total time derivative, and are thus physically equivalent.

To demonstrate how the diagrams at $\mathcal{O}\left(G v^{4}\right)$ are computed, we show how to calculate diagram $(b)$ in some detail. The first step is to form the expression of the diagram. This is achieved by coupling Eq. (17) to two copies of Eq. (7) for worldlines 1 and 2. The diagram is given as follows, where we have done the $\mathbf{q}$ integration to eliminate $\delta^{3}(\mathbf{p}+\mathbf{q})$,

$$
-i \int d t V=\frac{i m_{1} m_{2}}{8 m_{P l}^{2}} \int d t_{a} d t_{b} \int_{\mathbf{p}} \frac{e^{i \mathbf{p} \cdot\left(\mathbf{x}_{1}\left(t_{a}\right)-\mathbf{x}_{2}\left(t_{b}\right)\right)}}{\mathbf{p}^{4}} \frac{\left(1+\mathbf{v}_{1}\left(t_{a}\right)^{2}\right)\left(1+\mathbf{v}_{2}\left(t_{b}\right)^{2}\right)}{\left(1-\mathbf{v}_{1}\left(t_{a}\right)^{2}\right)^{1 / 2}\left(1-\mathbf{v}_{2}\left(t_{b}\right)^{2}\right)^{1 / 2}} \partial_{t_{a}} \partial_{t_{b}} \delta\left(t_{a}-t_{b}\right) .
$$

Care must be taken when labeling the time for each worldline Feynman rule, and here we have used $t_{a}$ and $t_{b}$. Since each worldline interaction is a different Feynman rule, it must therefore have a different dummy variable. Before explicitly using the time derivatives, we employ the Fourier integral from Eq. (13) to compute the remaining momentum integral. Now, an integration by parts on both time derivatives acting on $\delta\left(t_{a}-t_{b}\right)$ is required, which gives,

$$
-i \int d t V=\frac{-i G m_{1} m_{2}}{2} \int d t_{a} d t_{b} \delta\left(t_{a}-t_{b}\right)\left\{\partial_{t_{a}} \partial_{t_{b}}\left[\left|\mathbf{x}_{1}\left(t_{a}\right)-\mathbf{x}_{2}\left(t_{b}\right)\right| \frac{\left(1+\mathbf{v}_{1}\left(t_{a}\right)^{2}\right)\left(1+\mathbf{v}_{2}\left(t_{b}\right)^{2}\right)}{\left(1-\mathbf{v}_{1}\left(t_{a}\right)^{2}\right)^{1 / 2}\left(1-\mathbf{v}_{2}\left(t_{b}\right)^{2}\right)^{1 / 2}}\right]\right\} .
$$

At this stage, the time derivatives must be allowed to act on the square bracket. Only after this point can the $\delta\left(t_{a}-t_{b}\right)$ be used. It is clear that acceleration-dependent terms will arise. The potential contribution we obtain is exact to all orders in the PN expansion. Since we are only interested in the $2 \mathrm{PN}$ potential, we extract this piece from the result. For this purpose, we note that accelerations are power-counted as $a \sim v^{2} / r$. The final contribution, $V^{(b)}$, is given 


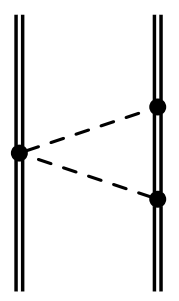

(g)

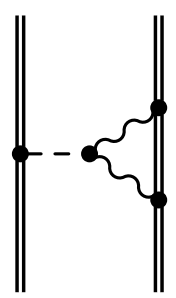

$(l)$

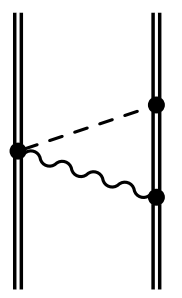

(h)

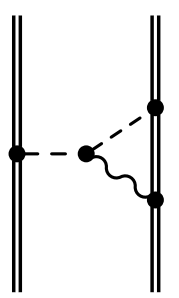

$(m)$

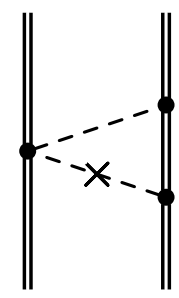

(i)

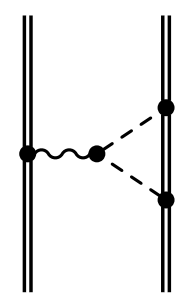

$(n)$

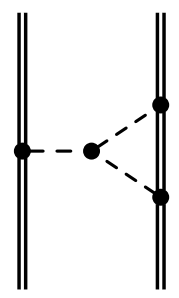

$(j)$

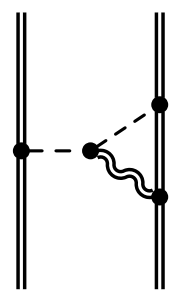

(o)

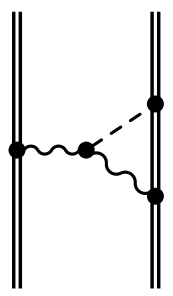

$(k)$

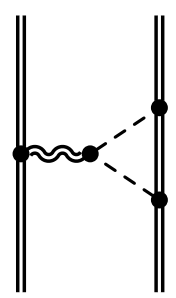

$(p)$

FIG. 5: Order $G^{2} v^{2}$ diagrams at $2 \mathrm{PN}$.

below, along with the other one-graviton exchange diagrams with propagator insertions at $\mathcal{O}\left(G v^{4}\right)$,

$$
\begin{aligned}
& V^{(b)}=-\frac{G m_{1} m_{2}}{r}\left(\frac{3}{4}\left(\mathbf{v}_{1} \cdot \mathbf{v}_{2}-\mathbf{n} \cdot \mathbf{v}_{1} \mathbf{n} \cdot \mathbf{v}_{2}\right)\left(\mathbf{v}_{1}^{2}+\mathbf{v}_{2}^{2}\right)\right) \\
& -G m_{1} m_{2}\left(\frac{3}{2} \mathbf{a}_{1} \cdot \mathbf{v}_{1} \mathbf{n} \cdot \mathbf{v}_{2}-\frac{3}{2} \mathbf{a}_{2} \cdot \mathbf{v}_{2} \mathbf{n} \cdot \mathbf{v}_{1}\right) \\
& V^{(c)}=-\frac{G m_{1} m_{2}}{r}\left(\frac{1}{8} \mathbf{v}_{1}^{2} \mathbf{v}_{2}^{2}+\frac{1}{4}\left(\mathbf{v}_{1} \cdot \mathbf{v}_{2}\right)^{2}+\frac{3}{8}\left(\mathbf{n} \cdot \mathbf{v}_{1}\right)^{2}\left(\mathbf{n} \cdot \mathbf{v}_{2}\right)^{2}\right. \\
& \left.-\frac{1}{2} \mathbf{v}_{1} \cdot \mathbf{v}_{2} \mathbf{n} \cdot \mathbf{v}_{1} \mathbf{n} \cdot \mathbf{v}_{2}-\frac{1}{8} \mathbf{v}_{1}^{2}\left(\mathbf{n} \cdot \mathbf{v}_{2}\right)^{2}-\frac{1}{8} \mathbf{v}_{2}^{2}\left(\mathbf{n} \cdot \mathbf{v}_{1}\right)^{2}\right) \\
& -G m_{1} m_{2}\left(\frac{1}{8} \mathbf{a}_{1} \cdot \mathbf{n}\left(\mathbf{v}_{2}^{2}-\left(\mathbf{n} \cdot \mathbf{v}_{2}\right)^{2}\right)-\frac{1}{8} \mathbf{a}_{2} \cdot \mathbf{n}\left(\mathbf{v}_{1}^{2}-\left(\mathbf{n} \cdot \mathbf{v}_{1}\right)^{2}\right)\right. \\
& \left.+\frac{1}{4} \mathbf{a}_{1} \cdot \mathbf{v}_{2} \mathbf{n} \cdot \mathbf{v}_{2}-\frac{1}{4} \mathbf{a}_{2} \cdot \mathbf{v}_{1} \mathbf{n} \cdot \mathbf{v}_{1}\right) \\
& -G m_{1} m_{2} r\left(-\frac{1}{8} \mathbf{a}_{1} \cdot \mathbf{a}_{2}-\frac{1}{8} \mathbf{a}_{1} \cdot \mathbf{n} \mathbf{a}_{2} \cdot \mathbf{n}\right) \\
& V^{(e)}=-\frac{G m_{1} m_{2}}{r}\left(-2\left(\mathbf{v}_{1} \cdot \mathbf{v}_{2}\right)^{2}+2 \mathbf{v}_{1} \cdot \mathbf{v}_{2} \mathbf{n} \cdot \mathbf{v}_{1} \mathbf{n} \cdot \mathbf{v}_{2}\right) \\
& -G m_{1} m_{2}\left(-2 \mathbf{a}_{1} \cdot \mathbf{v}_{2} \mathbf{n} \cdot \mathbf{v}_{2}+2 \mathbf{a}_{2} \cdot \mathbf{v}_{1} \mathbf{n} \cdot \mathbf{v}_{1}\right) \\
& -G m_{1} m_{2} r\left(2 \mathbf{a}_{1} \cdot \mathbf{a}_{2}\right) \text {. }
\end{aligned}
$$

Note that all the potential contributions $V^{(b)}, V^{(c)}$, and $V^{(e)}$, contain acceleration-dependent terms. They are generated from either a time derivative acting on a worldline velocity factor or from two time derivatives acting on $\mathbf{x}_{1}$ or $\mathbf{x}_{2}$.

\section{B. Order $G^{2} v^{2}$ diagrams}

The first three diagrams at $\mathcal{O}\left(G^{2} v^{2}\right),(g),(h)$, and $(i)$ of Fig. [5, are obtained by modifying the $\mathcal{O}(G)$ topology through the addition of one extra gravitational leg connecting the two worldlines. As we will see, such diagrams, where a topology of lower order in $G$ is augmented by an additional leg between the worldlines, are particularly 
simple to compute because their expressions factorize. To compute these diagrams we need two additional Feynman rules for the worldline couplings. The first has two $\phi$ fields coupling to the worldline, and the second has a $\phi$ and an $A_{i}$ coupling to the worldline. These are respectively,

$$
\begin{aligned}
& \|-=-\frac{i m}{m_{P l}^{2}} \int d t \int_{\mathbf{k}, \mathbf{q}} e^{i(\mathbf{k}+\mathbf{q}) \cdot \times} \frac{1-6 \mathbf{v}^{2}+\mathbf{v}^{4}}{\left(1-\mathbf{v}^{2}\right)^{3 / 2}} \\
& \sim \sim-\frac{i m}{m_{P l}^{2}} \int d t \int_{\mathbf{k}, \mathbf{q}} e^{i(\mathbf{k}+\mathbf{q}) \cdot \mathbf{x}} \frac{\left(1-3 \mathbf{v}^{2}\right) \mathbf{v}_{i}}{\left(1-\mathbf{v}^{2}\right)^{3 / 2}}
\end{aligned}
$$

These rules are again exact in the orbital velocity $v$.

We demonstrate how to calculate the three diagrams $(g),(h)$, and $(i)$, by considering diagram $(h)$. One can write this diagram by using Eqs. (7), (8), and (26) for the worldline couplings, and Eqs. (10) and (11) for the propagators. The diagram is

$$
\begin{aligned}
-i \int d t V= & \frac{i m_{1} m_{2}^{2}}{16 m_{P l}^{4}} \int d t_{a} d t_{b} d t_{c} \delta\left(t_{a}-t_{b}\right) \delta\left(t_{a}-t_{c}\right) \int_{\mathbf{k}, \mathbf{l}, \mathbf{p}, \mathbf{q}} e^{i\left[(\mathbf{k}+\mathbf{p}) \cdot \mathbf{x}_{1}\left(t_{a}\right)+\mathbf{l} \cdot \mathbf{x}_{2}\left(t_{b}\right)+\mathbf{q} \cdot \mathbf{x}_{2}\left(t_{c}\right)\right]} \\
& \times \frac{(2 \pi)^{3} \delta^{3}(\mathbf{k}+\mathbf{l})(2 \pi)^{3} \delta^{3}(\mathbf{p}+\mathbf{q})}{\mathbf{k}^{2} \mathbf{p}^{2}} \frac{\mathbf{v}_{1}\left(t_{a}\right) \cdot \mathbf{v}_{2}\left(t_{c}\right)\left(1-3 \mathbf{v}_{1}\left(t_{a}\right)^{2}\right)\left(1+\mathbf{v}_{2}\left(t_{b}\right)^{2}\right)}{\left(1-\mathbf{v}_{1}\left(t_{a}\right)^{2}\right)^{3 / 2}\left(1-\mathbf{v}_{2}\left(t_{b}\right)^{2}\right)^{1 / 2}\left(1-\mathbf{v}_{2}\left(t_{c}\right)^{2}\right)^{1 / 2}}
\end{aligned}
$$

The symmetry factor of this diagram is 1 . Since there are no time derivatives in this expression, the time delta functions can be used immediately. We can also use the three-momentum delta functions; doing so leads to

$$
-i \int d t V=\frac{i m_{1} m_{2}^{2}}{16 m_{P l}^{4}} \int d t \int_{\mathbf{k}, \mathbf{p}} \frac{e^{i \mathbf{k} \cdot \mathbf{r}}}{\mathbf{k}^{2}} \frac{e^{i \mathbf{p} \cdot \mathbf{r}}}{\mathbf{p}^{2}} \frac{\mathbf{v}_{1} \cdot \mathbf{v}_{2}\left(1-3 \mathbf{v}_{1}^{2}\right)\left(1+\mathbf{v}_{2}^{2}\right)}{\left(1-\mathbf{v}_{1}^{2}\right)^{3 / 2}\left(1-\mathbf{v}_{2}^{2}\right)}
$$

Looking at this equation, it is clear that the two momentum integrations factorize into two Fourier transforms. They are seen to be the same in this diagram, and we can simply use the Fourier integral, Eq. (13). Our final result for the exact potential contribution from diagram $(h)$ to all orders in the PN expansion, is then

$$
V_{\text {Exact }}^{(h)}=\frac{-4 G^{2} m_{1} m_{2}^{2}}{r^{2}} \frac{\mathbf{v}_{1} \cdot \mathbf{v}_{2}\left(1-3 \mathbf{v}_{1}^{2}\right)\left(1+\mathbf{v}_{2}^{2}\right)}{\left(1-\mathbf{v}_{1}^{2}\right)^{3 / 2}\left(1-\mathbf{v}_{2}^{2}\right)} .
$$

The calculation of the other two diagrams with this topology, diagrams $(g)$ and $(i)$, proceed similarly. However, when propagator insertions are present, as in diagram $(i)$, the time delta functions cannot be used immediately. One must first integrate by parts, and act with the time derivatives, before using the time delta functions. The procedure is analogous to diagram $(b)$ in Sec. [II A At $2 \mathrm{PN}$, we have the following contributions to the potential:

$$
\begin{aligned}
V^{(g)} & =\frac{G^{2} m_{1} m_{2}^{2}}{r^{2}}\left(-\frac{9}{4} \mathbf{v}_{1}^{2}+\frac{3}{2} \mathbf{v}_{2}^{2}\right) \\
V^{(h)} & =\frac{G^{2} m_{1} m_{2}^{2}}{r^{2}}\left(-4 \mathbf{v}_{1} \cdot \mathbf{v}_{2}\right) \\
V^{(i)} & =\frac{G^{2} m_{1} m_{2}^{2}}{r^{2}}\left(\frac{1}{2}\left(\mathbf{n} \cdot \mathbf{v}_{2}\right)^{2}+\frac{1}{2} \mathbf{v}_{1} \cdot \mathbf{v}_{2}-\mathbf{n} \cdot \mathbf{v}_{1} \mathbf{n} \cdot \mathbf{v}_{2}\right) .
\end{aligned}
$$

Although we do not display the diagrams where the two worldlines are interchanged, they must be accounted for in the final 2PN Lagrangian.

We now proceed to the diagrams where the topology is that of Fig. 2(b), where a three-graviton vertex mediates the interaction. First, the vertices for the gravitational self-interactions must be derived, and this is done in a straightforward way from the sum of the Einstein-Hilbert action, Eq. (11), and the gauge fixing action, Eq. (2). At 
$2 \mathrm{PN}$, the KS parametrization simplifies the vertex derivations, since we need at most one leg to be the tensor field $\sigma_{i j}$. Once the appropriate terms in the action are established via power-counting factors of $v$, the Feynman rule for the vertex is computed. Then each leg of a given vertex is multiplied by the appropriate propagator to obtain the three-point function. For diagrams $(j),(k),(l),(m),(n),(o)$, and $(p)$ of Fig. 5 , we use the following three-point functions:

$$
\begin{aligned}
\left\langle T \phi_{a} \phi_{b} \phi_{c}\right\rangle=\frac{1}{16 m_{P l}}\left\{\left(\partial_{t_{a}} \delta\left(t_{a}-t_{c}\right)\right)\left(\partial_{t_{b}} \delta\left(t_{b}-t_{c}\right)\right)+\left(\partial_{t_{a}} \delta\left(t_{a}-t_{b}\right)\right)\left(\partial_{t_{c}} \delta\left(t_{c}-t_{b}\right)\right)\right. \\
\left.+\left(\partial_{t_{b}} \delta\left(t_{b}-t_{a}\right)\right)\left(\partial_{t_{c}} \delta\left(t_{c}-t_{a}\right)\right)\right\} \times \frac{1}{\mathbf{k}_{a}^{2} \mathbf{k}_{b}^{2} \mathbf{k}_{c}^{2}}(2 \pi)^{3} \delta^{3}\left(\mathbf{k}_{a}+\mathbf{k}_{b}+\mathbf{k}_{c}\right) \\
\left\langle T \phi_{a} \phi_{b} A_{c}^{i}\right\rangle=-\frac{i}{16 m_{P l}}\left\{\left(\mathbf{k}_{b}^{i} \partial_{t_{a}}+\mathbf{k}_{a}^{i} \partial_{t_{b}}\right) \delta\left(t_{a}-t_{c}\right) \delta\left(t_{b}-t_{c}\right)\right\} \times \frac{1}{\mathbf{k}_{a}^{2} \mathbf{k}_{b}^{2} \mathbf{k}_{c}^{2}}(2 \pi)^{3} \delta^{3}\left(\mathbf{k}_{a}+\mathbf{k}_{b}+\mathbf{k}_{c}\right) \\
\left\langle T \phi_{a} A_{b}^{i} A_{c}^{j}\right\rangle=\frac{1}{4 m_{P l}} \delta\left(t_{a}-t_{c}\right) \delta\left(t_{b}-t_{c}\right) \times \frac{\mathbf{k}_{b}^{i} \mathbf{k}_{c}^{j}-\mathbf{k}_{c}^{i} \mathbf{k}_{b}^{j}+\mathbf{k}_{b} \cdot \mathbf{k}_{c} \delta^{i j}}{\mathbf{k}_{a}^{2} \mathbf{k}_{b}^{2} \mathbf{k}_{c}^{2}}(2 \pi)^{3} \delta^{3}\left(\mathbf{k}_{a}+\mathbf{k}_{b}+\mathbf{k}_{c}\right) \\
\left\langle T \phi_{a} \phi_{b} \sigma_{c}^{i j}\right\rangle=\frac{1}{16 m_{P l}} \delta\left(t_{a}-t_{c}\right) \delta\left(t_{b}-t_{c}\right) \times \frac{\mathbf{k}_{a}^{i} \mathbf{k}_{b}^{j}+\mathbf{k}_{b}^{i} \mathbf{k}_{a}^{j}}{\mathbf{k}_{a}^{2} \mathbf{k}_{b}^{2} \mathbf{k}_{c}^{2}}(2 \pi)^{3} \delta^{3}\left(\mathbf{k}_{a}+\mathbf{k}_{b}+\mathbf{k}_{c}\right),
\end{aligned}
$$

where the lower index on a field denotes its dependence on $t$ and $\mathbf{k}$.

Because of the nonlinear structure of the diagrams $(j)$ through $(p)$, their contributions do not factorize, and we have to calculate an integral which corresponds to a one-loop Feynman integral. To demonstrate the calculation of this class of diagrams, we consider diagram $(k)$. This diagram uses the three-point function $\left\langle T \phi_{a} A_{b}^{i} A_{c}^{j}\right\rangle$ from Eq. (35). The diagram is formed by coupling Eq. (35) to the worldlines using Eqs. (7) and (8) and the symmetry factor of this diagram is 1 . Given there are no time derivatives, we are free to do two of the time integrations immediately. We also do the integration over the three-momentum which labels the $\phi$ leg. The result of these operations is

$$
-i \int d t V=\frac{i m_{1} m_{2}^{2}}{4 m_{P l}^{4}} \int d t \frac{\left(1+\mathbf{v}_{2}^{2}\right) \mathbf{v}_{1}^{i} \mathbf{v}_{2}^{j}}{\left(1-\mathbf{v}_{1}^{2}\right)^{1 / 2}\left(1-\mathbf{v}_{2}^{2}\right)} I^{i j}(\mathbf{r})
$$

where the integral $I^{i j}(\mathbf{r})$ is given by

$$
I^{i j}(\mathbf{r})=\int_{\mathbf{p}, \mathbf{q}} e^{i \mathbf{p} \cdot \mathbf{r}} \frac{\mathbf{p}^{i} \mathbf{q}^{j}-\mathbf{q}^{i} \mathbf{p}^{j}+\mathbf{p} \cdot \mathbf{q} \delta^{i j}}{\mathbf{p}^{2} \mathbf{q}^{2}(\mathbf{p}+\mathbf{q})^{2}}=\int_{\mathbf{p}} e^{i \mathbf{p} \cdot \mathbf{r}} \frac{\mathbf{p}^{i} \delta^{j a}-\mathbf{p}^{j} \delta^{i a}+\mathbf{p}^{a} \delta^{i j}}{\mathbf{p}^{2}} \int_{\mathbf{q}} \frac{\mathbf{q}^{a}}{\mathbf{q}^{2}(\mathbf{q}+\mathbf{p})^{2}} .
$$

The next step is to evaluate the momentum integral, $I^{i j}(\mathbf{r})$. From Eq. (38) it is apparent that $I^{i j}(\mathbf{r})$ is calculated by first computing the q-integration which corresponds to a one-loop Feynman integral and subsequently performing a Fourier integration over p. The Feynman integral is of vector nature, and we reduce it to a scalar integral in the usual way by noting that its expression must be proportional to $\mathbf{p}^{a}$. The scalar integral is then computed in dimensional regularization using the $d$-dimensional master integral

$$
\int \frac{d^{d} \mathbf{k}}{(2 \pi)^{d}} \frac{1}{\left[(\mathbf{k}+\mathbf{p})^{2}\right]^{n_{1}}\left[\mathbf{k}^{2}\right]^{n_{2}}}=\frac{1}{(4 \pi)^{d / 2}} \frac{\Gamma\left(n_{1}+n_{2}-d / 2\right)}{\Gamma\left(n_{1}\right) \Gamma\left(n_{2}\right)} \frac{\Gamma\left(d / 2-n_{1}\right) \Gamma\left(d / 2-n_{2}\right)}{\Gamma\left(d-n_{1}-n_{2}\right)}\left(\mathbf{p}^{2}\right)^{d / 2-n_{1}-n_{2}} .
$$

The resulting expression for the integral $I^{i j}(\mathbf{r})$ then becomes

$$
I^{i j}(\mathbf{r})=-\frac{\delta^{i j}}{16} \int_{\mathbf{p}} e^{i \mathbf{p} \cdot \mathbf{r}} \frac{1}{|\mathbf{p}|}
$$

and the remaining Fourier transform integral is performed with Eq. (13). Putting all terms back together gives the exact potential for diagram $(k)$,

$$
V_{\text {Exact }}^{(k)}=\frac{G^{2} m_{1} m_{2}^{2}}{r^{2}} \frac{8 \mathbf{v}_{1} \cdot \mathbf{v}_{2}\left(1+\mathbf{v}_{2}^{2}\right)}{\left(1-\mathbf{v}_{1}^{2}\right)^{1 / 2}\left(1-\mathbf{v}_{2}^{2}\right)} .
$$

The $2 \mathrm{PN}$ piece of this potential is extracted below. 


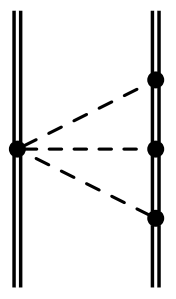

$(q)$

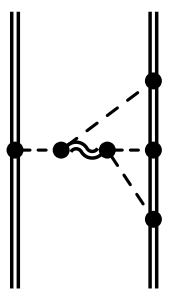

$(r)$

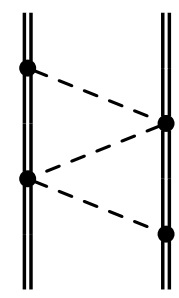

$(s)$

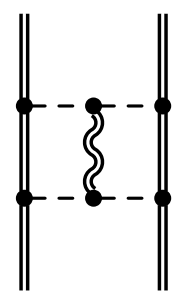

$(t)$

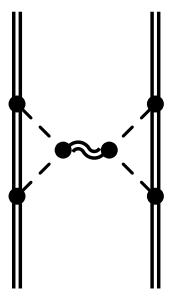

$(u)$

FIG. 6: Order $G^{3} v^{0}$ diagrams at $2 \mathrm{PN}$.

The remaining diagrams from $(j)$ through $(p)$ are all evaluated using the same methods as explained for diagram $(k)$. The only complication are the time derivatives which occur in the three-point functions $\left\langle T \phi_{a} \phi_{b} \phi_{c}\right\rangle$ and $\left\langle T \phi_{a} \phi_{b} A_{c}^{i}\right\rangle$, Eqs. (33) and (34), respectively. So when calculating the diagrams $(j),(m)$, and $(n)$, it is necessary to integrate by parts as explained in Sec. IIIA We use Eq. (39) (and its vector or tensor extensions) for any one-loop integrals, and Eq. (13) for the Fourier transforms. Additionally, for some diagrams we need vector and tensor Fourier integrals which are obtained from the scalar Fourier integral in Eq. (13) by taking derivatives with respect to $\mathbf{r}$. At $\mathcal{O}\left(G^{2} v^{2}\right)$, the contributions from the individual diagrams with the topology Fig. 2(b) are

$$
\begin{aligned}
V^{(j)} & =\frac{G^{2} m_{1} m_{2}^{2}}{r^{2}}\left(\frac{1}{2} \mathbf{v}_{2}^{2}-\frac{1}{2}\left(\mathbf{n} \cdot \mathbf{v}_{2}\right)^{2}-2 \mathbf{v}_{1} \cdot \mathbf{v}_{2}+4 \mathbf{n} \cdot \mathbf{v}_{1} \mathbf{n} \cdot \mathbf{v}_{2}\right) \\
V^{(k)} & =\frac{G^{2} m_{1} m_{2}^{2}}{r^{2}}\left(8 \mathbf{v}_{1} \cdot \mathbf{v}_{2}\right) \\
V^{(l)} & =\frac{G^{2} m_{1} m_{2}^{2}}{r^{2}}\left(-4 \mathbf{v}_{2}^{2}\right) \\
V^{(m)} & =\frac{G^{2} m_{1} m_{2}^{2}}{r^{2}}\left(2 \mathbf{v}_{2}^{2}-4\left(\mathbf{n} \cdot \mathbf{v}_{2}\right)^{2}+2 \mathbf{v}_{1} \cdot \mathbf{v}_{2}-4 \mathbf{n} \cdot \mathbf{v}_{1} \mathbf{n} \cdot \mathbf{v}_{2}\right) \\
V^{(n)} & =\frac{G^{2} m_{1} m_{2}^{2}}{r^{2}}\left(-\mathbf{v}_{1} \cdot \mathbf{v}_{2}+\mathbf{n} \cdot \mathbf{v}_{1} \mathbf{n} \cdot \mathbf{v}_{2}\right) \\
V^{(o)} & =\frac{G^{2} m_{1} m_{2}^{2}}{r^{2}}\left(-2 \mathbf{v}_{2}^{2}+4\left(\mathbf{n} \cdot \mathbf{v}_{2}\right)^{2}\right) \\
V^{(p)} & =\frac{G^{2} m_{1} m_{2}^{2}}{r^{2}}\left(\frac{1}{2} \mathbf{v}_{1}^{2}-\frac{1}{2}\left(\mathbf{n} \cdot \mathbf{v}_{1}\right)^{2}\right) .
\end{aligned}
$$

All of the above contributions with $(1 \leftrightarrow 2)$ must be added to the final potential in order to account for the diagrams with the two worldlines interchanged.

\section{Order $G^{3} v^{0}$ diagrams}

Finally, we progress onto the diagrams at $\mathcal{O}\left(G^{3} v^{0}\right)$. The diagrams which must be evaluated are $(q),(r),(s),(t)$, and $(u)$, as shown in Fig. 6 For diagram $(q)$ a new worldline vertex will be required coupling three $\phi$ fields to the worldline at one point. The Feynman rule is

$$
\|=-\frac{i m}{m_{P l}^{3}} \int d t \int_{\mathbf{k}, \mathbf{q}, \mathbf{p}} e^{i(\mathbf{k}+\mathbf{q}+\mathbf{p}) \cdot \mathbf{x}} \frac{1+11 \mathbf{v}^{2}+11 \mathbf{v}^{4}+\mathbf{v}^{6}}{\left(1-\mathbf{v}^{2}\right)^{5 / 2}}
$$

which is exact to all orders in $v$. At this order, the potential contribution at $2 \mathrm{PN}$ is static and there are no velocity factors in the final potential contributions. Given this is the case, we do not keep any velocity factors from the worldline vertices when we discuss the calculations below.

The two simple diagrams at this order are $(q)$ and $(s)$. These diagrams are easy because there are no internal vertices - only propagators and worldline couplings. The diagrams therefore factor into simple Fourier transforms, 
which are evaluated using Eq. (13). This is analogous to the evaluation of diagram $(h)$ in Sec. 【IIB. The symmetry factors are $1 / 6$ and 1 for $(q)$ and $(s)$, respectively. Computing each diagram gives the following potential contributions:

$$
\begin{aligned}
V^{(q)} & =-\frac{G^{3} m_{1} m_{2}^{3}}{6 r^{3}} \\
V^{(s)} & =-\frac{G^{3} m_{1}^{2} m_{2}^{2}}{r^{3}},
\end{aligned}
$$

where the contribution of diagram $(q)$ must be added with $(1 \leftrightarrow 2)$ in the final potential.

The next set of diagrams at $\mathcal{O}\left(G^{3} v^{0}\right)$ are no longer as simple as $(q)$ and $(s)$. This is due to the presence of the four-point function, which is used to construct the diagrams $(r),(t)$, and $(u)$. The only four-point function needed at $2 \mathrm{PN}$ is derived by joining two $\phi \phi \sigma_{i j}$ vertices with an intermediate $\sigma_{i j}$ propagator, Eq. (12), and attaching $\phi$ propagators, given in Eq. (10), to the external legs. This is given by

$$
\begin{aligned}
\langle T \underbrace{\phi_{a} \phi_{b}} \underbrace{\phi_{c} \phi_{d}}\rangle= & \frac{i}{128 m_{P l}^{2}} \delta\left(t_{a}-t_{d}\right) \delta\left(t_{b}-t_{d}\right) \delta\left(t_{c}-t_{d}\right) \\
& \times \frac{\mathbf{k}_{a} \cdot \mathbf{k}_{c} \mathbf{k}_{b} \cdot \mathbf{k}_{d}+\mathbf{k}_{a} \cdot \mathbf{k}_{d} \mathbf{k}_{b} \cdot \mathbf{k}_{c}-\mathbf{k}_{a} \cdot \mathbf{k}_{b} \mathbf{k}_{c} \cdot \mathbf{k}_{d}}{\left(\mathbf{k}_{a}+\mathbf{k}_{b}\right)^{2} \mathbf{k}_{a}^{2} \mathbf{k}_{b}^{2} \mathbf{k}_{c}^{2} \mathbf{k}_{d}^{2}}(2 \pi)^{3} \delta^{3}\left(\mathbf{k}_{a}+\mathbf{k}_{b}+\mathbf{k}_{c}+\mathbf{k}_{d}\right),
\end{aligned}
$$

where each set of two $\phi$ fields connected by a brace have the same intermediate $\phi \phi \sigma_{i j}$ vertex.

Of the three diagrams left to compute, $(t)$ is the most involved, so we will compute it explicitly. We first note that the symmetry factor of this diagram is $1 / 2$. The diagram is constructed in the usual manner, using Eq. (17) and Eq. (52), but we will only work to $\mathcal{O}\left(G^{3} v^{0}\right)$ in what follows neglecting all velocity factors of the vertices. First, we integrate over all the time delta functions. Then, we relabel the three-momentum of the four-point function as $\left(\mathbf{k}_{a}, \mathbf{k}_{b}, \mathbf{k}_{c}, \mathbf{k}_{d}\right) \rightarrow(\mathbf{k}, \mathbf{q}, \mathbf{p}, \mathbf{l})$ and couple $\mathbf{k}$ and $\mathbf{p}$ to worldline 1 and $\mathbf{q}$ and $\mathbf{l}$ to worldline 2 . The $\mathbf{l}$ integration is then performed using the $\delta$-function, which takes $\mathbf{l} \rightarrow-(\mathbf{k}+\mathbf{p}+\mathbf{q})$. After some algebra, redefining of $\mathbf{k} \rightarrow \mathbf{k}+\mathbf{p}$ is seen to be useful. This gives the following expression:

$$
-i \int d t V=\frac{i m_{1}^{2} m_{2}^{2}}{256 m_{P l}^{6}} \int d t \int_{\mathbf{k}} e^{i \mathbf{k} \cdot \mathbf{r}}\left(I_{\mathrm{I}}(\mathbf{k})+I_{\mathrm{II}}(\mathbf{k})+I_{\mathrm{III}}(\mathbf{k})+I_{\mathrm{IV}}(\mathbf{k})\right)
$$

where the terms $I_{\mathrm{I}}(\mathbf{k}), I_{\mathrm{II}}(\mathbf{k}), I_{\mathrm{III}}(\mathbf{k})$, and $I_{\mathrm{IV}}(\mathbf{k})$ are the integrals

$$
\begin{aligned}
I_{\mathrm{I}}(\mathbf{k}) & =\int_{\mathbf{p}, \mathbf{q}}-\mathbf{k} \cdot \mathbf{p q}^{2} / Q(\mathbf{k}, \mathbf{p}, \mathbf{q}) \\
I_{\mathrm{II}}(\mathbf{k}) & =\int_{\mathbf{p}, \mathbf{q}} \mathbf{k} \cdot \mathbf{q p}^{2} / Q(\mathbf{k}, \mathbf{p}, \mathbf{q}) \\
I_{\mathrm{III}}(\mathbf{k}) & =\int_{\mathbf{p}, \mathbf{q}} \mathbf{p}^{2} \mathbf{q}^{2} / Q(\mathbf{k}, \mathbf{p}, \mathbf{q}) \\
I_{\mathrm{IV}}(\mathbf{k}) & =\int_{\mathbf{p}, \mathbf{q}}-\mathbf{k}^{2} \mathbf{p} \cdot \mathbf{q} / Q(\mathbf{k}, \mathbf{p}, \mathbf{q}),
\end{aligned}
$$

and $Q(\mathbf{k}, \mathbf{p}, \mathbf{q})=(\mathbf{k}+\mathbf{q})^{2}(\mathbf{k}-\mathbf{p})^{2}(\mathbf{k}+\mathbf{q}-\mathbf{p})^{2} \mathbf{q}^{2} \mathbf{p}^{2}$. These integrals correspond to two-loop Feynman integrals, and we will show how to compute them efficiently by reducing them to the one-loop master integral in Eq. (39).

The first three integrals $I_{\mathrm{I}}(\mathbf{k}), I_{\mathrm{II}}(\mathbf{k})$, and $I_{\mathrm{III}}(\mathbf{k})$ can be computed straightforwardly using the $d$-dimensional oneloop scalar integral from Eq. (39) twice, on both the $\mathbf{p}$ and $\mathbf{q}$ integrations. One can see that $I_{\mathrm{I}}(\mathbf{k})=I_{\mathrm{II}}(\mathbf{k})$ by making the replacement $(\mathbf{p}, \mathbf{q}) \rightarrow(-\mathbf{q},-\mathbf{p})$, in $I_{\mathrm{II}}(\mathbf{k})$. The reason why one can evaluate these two-loop integrals employing solely a single one-loop master integral, is that these integrals correspond to nested loop diagrams. Here, it is important to keep the dimension $d$ of the integrals arbitrary and only set $d=3$ after the final Fourier integration over $\mathbf{k}$ has been performed.

The remaining two-loop integral, $I_{\mathrm{IV}}(\mathbf{k})$, requires an integration by parts trick [41] in order to reduce it in terms of our master integral of Eq. (39). But first, we redefine $\mathbf{q} \rightarrow \mathbf{q}+\mathbf{k}$. This allows us to split the integral into four terms, three of which can be evaluated using same methods as discussed in the previous paragraph. The remaining term is a two-loop integral with five factors in the denominator

$$
\frac{\mathbf{k}^{4}}{2} \int_{\mathbf{p}, \mathbf{q}} \frac{1}{\mathbf{q}^{2}(\mathbf{q}-\mathbf{k})^{2}(\mathbf{q}+\mathbf{p})^{2}(\mathbf{p}+\mathbf{k})^{2} \mathbf{p}^{2}}
$$


Using the integration by parts trick we can write this integral as

$$
\int_{\mathbf{p}, \mathbf{q}} \frac{1}{\mathbf{q}^{2}(\mathbf{q}-\mathbf{k})^{2}(\mathbf{q}+\mathbf{p})^{2}(\mathbf{p}+\mathbf{k})^{2} \mathbf{p}^{2}}=\frac{2}{d-4} \int_{\mathbf{p}, \mathbf{q}}\left(\frac{1}{\mathbf{p}^{2} \mathbf{q}^{2}(\mathbf{q}+\mathbf{k})^{2}(\mathbf{p}+\mathbf{k})^{4}}-\frac{1}{\mathbf{p}^{2} \mathbf{q}^{2}(\mathbf{p}+\mathbf{q})^{2}(\mathbf{p}+\mathbf{k})^{4}}\right) .
$$

Once in this form, we can evaluate it using Eq. (39) twice.

After all the Feynman integrals have been evaluated, we perform the remaining Fourier transformation integral using Eq. (13). Subsequently, we can set $d=3$ and find a contribution to the potential

$$
V^{(t)}=-\frac{2 G^{3} m_{1}^{2} m_{2}^{2}}{r^{3}}
$$

The remaining two diagrams, $(r)$ and $(u)$, are simpler to compute. They are evaluated with the methods we have discussed, where once again it is necessary to compute two-loop integrals by applying the master integral Eq. (39) to two integrations. The results of the calculations are

$$
\begin{aligned}
& V^{(r)}=-\frac{G^{3} m_{1} m_{2}^{3}}{3 r^{3}} \\
& V^{(u)}=0,
\end{aligned}
$$

and the contribution of the potential $V^{(r)}$ must be added with $(1 \leftrightarrow 2)$ in the final potential. It is worth noting that $V^{(u)}=0$ because the diagram gives a purely short distance contribution $V \sim \delta(r)$, which we have dropped.

\section{RESULTS}

Having computed all the diagrams, we can now construct the $2 \mathrm{PN}$ interaction Lagrangian. There are two contributions which we need. The first is the kinetic energy, which is obtained by expanding the matter coupling action, Eq. (3), to $\mathcal{O}\left(v^{6}\right)$ while setting all fields to zero. The final potential comes by summing each potential contribution from the diagrams, $V^{(a)}$ to $V^{(u)}$. At this stage, we add all contributions with the worldlines interchanged, as appropriate. Our 2PN interaction Lagrangian for a binary system is then given by

$$
\begin{aligned}
L_{2 P N}= & \frac{m_{1} \mathbf{v}_{1}^{6}}{16} \\
+ & \frac{G m_{1} m_{2}}{r}\left(\frac{7}{8} \mathbf{v}_{1}^{4}-\frac{5}{4} \mathbf{v}_{1}^{2} \mathbf{v}_{1} \cdot \mathbf{v}_{2}-\frac{3}{4} \mathbf{v}_{1}^{2} \mathbf{n} \cdot \mathbf{v}_{1} \mathbf{n} \cdot \mathbf{v}_{2}+\frac{3}{16} \mathbf{v}_{1}^{2} \mathbf{v}_{2}^{2}+\frac{1}{8}\left(\mathbf{v}_{1} \cdot \mathbf{v}_{2}\right)^{2}\right. \\
& \left.\quad-\frac{1}{8} \mathbf{v}_{1}^{2}\left(\mathbf{n} \cdot \mathbf{v}_{2}\right)^{2}+\frac{3}{4} \mathbf{n} \cdot \mathbf{v}_{1} \mathbf{n} \cdot \mathbf{v}_{2} \mathbf{v}_{1} \cdot \mathbf{v}_{2}+\frac{3}{16}\left(\mathbf{n} \cdot \mathbf{v}_{1}\right)^{2}\left(\mathbf{n} \cdot \mathbf{v}_{2}\right)^{2}\right) \\
+ & G m_{1} m_{2}\left(\frac{1}{8} \mathbf{a}_{1} \cdot \mathbf{n} \mathbf{v}_{2}^{2}+\frac{3}{2} \mathbf{a}_{1} \cdot \mathbf{v}_{1} \mathbf{n} \cdot \mathbf{v}_{2}-\frac{7}{4} \mathbf{a}_{1} \cdot \mathbf{v}_{2} \mathbf{n} \cdot \mathbf{v}_{2}-\frac{1}{8} \mathbf{a}_{1} \cdot \mathbf{n}\left(\mathbf{n} \cdot \mathbf{v}_{2}\right)^{2}\right) \\
+ & G m_{1} m_{2} r\left(\frac{15}{16} \mathbf{a}_{1} \cdot \mathbf{a}_{2}-\frac{1}{16} \mathbf{a}_{1} \cdot \mathbf{n} \mathbf{a}_{2} \cdot \mathbf{n}\right) \\
+ & \frac{G^{2} m_{1} m_{2}^{2}}{r^{2}}\left(\frac{7}{4} \mathbf{v}_{1}^{2}+2 \mathbf{v}_{2}^{2}-\frac{7}{2} \mathbf{v}_{1} \cdot \mathbf{v}_{2}+\frac{1}{2}\left(\mathbf{n} \cdot \mathbf{v}_{1}\right)^{2}\right) \\
+ & \frac{G^{3} m_{1} m_{2}^{3}}{2 r^{3}}+\frac{3 G^{3} m_{1}^{2} m_{2}^{2}}{2 r^{3}}+(1 \leftrightarrow 2),
\end{aligned}
$$

where $(1 \leftrightarrow 2)$ refers to all terms given previously, with the labels 1 and 2 interchanged. Note that $\mathbf{n} \rightarrow-\mathbf{n}$ under this exchange.

Upon comparison to the $2 \mathrm{PN}$ piece of the Lagrangian in Eq. (174) in [14], it is apparent that our Lagrangian does not have the same form. However, when the equations of motion (EOM) are computed we recover the same $2 \mathrm{PN}$ EOM as in [14]. This shows the physical equivalence of our Lagrangian and the one in [14]. At the level of the 
Lagrangian, this can be shown directly by relating our result in harmonic gauge to the standard 2PN Lagrangian in harmonic gauge of [14] via a total derivative and two double zero terms [42]. A double zero term is an expression which vanishes at $2 \mathrm{PN}$ by the use of the lower order EOM. Adding such a term modifies the form of the Lagrangian, but does not change the EOM or the choice of gauge. The terms we add are given by

$$
\begin{aligned}
& \delta L_{1}=\frac{1}{8} \frac{G m_{1} m_{2}}{r}\left(\mathbf{r} \cdot \mathbf{a}_{1}+\frac{G m_{2}}{r}\right)\left(\mathbf{r} \cdot \mathbf{a}_{2}-\frac{G m_{1}}{r}\right) \\
& \delta L_{2}=-\frac{15}{8} G m_{1} m_{2} r\left(\mathbf{a}_{1}^{i}+\frac{G m_{2}}{r^{3}} \mathbf{r}^{i}\right)\left(\mathbf{a}_{2}^{i}-\frac{G m_{1}}{r^{3}} \mathbf{r}^{i}\right) \\
& \delta L_{3}=\frac{d}{d t}\left[\frac{7}{4} \frac{G^{2} m_{1} m_{2}}{r^{2}}\left(m_{2} \mathbf{r} \cdot \mathbf{v}_{2}-m_{1} \mathbf{r} \cdot \mathbf{v}_{1}\right)+\frac{3}{4} \frac{G m_{1} m_{2}}{r}\left(\mathbf{r} \cdot \mathbf{v}_{1} \mathbf{v}_{2}^{2}-\mathbf{r} \cdot \mathbf{v}_{2} \mathbf{v}_{1}^{2}\right)\right],
\end{aligned}
$$

where the first two terms are double zero terms and the last one is a total time derivative. By adding these terms to our Lagrangian at 2PN, Eq. (63), we obtain the Lagrangian in 14]. We again emphasize that applying these transformations leaves the EOM unchanged, so that we remain in harmonic gauge.

We have also calculated the observable $E(\omega)$ for a circular orbit and recover the known $2 \mathrm{PN}$ result [14]. We note that if the accelerations were replaced in our Lagrangian, using the LO EOM, $E(\omega)$ is unchanged, although all intermediate expressions are different. This is because $E(\omega)$ is physical and therefore gauge invariant, and using the EOM at the level of the Lagrangian corresponds to a change in gauge.

\section{CONCLUSION}

We have demonstrated how to use the EFT method [19] to efficiently calculate the next-to-next-to-leading order Lagrangian describing the conservative dynamics of a binary system. We have shown how to systematically determine all Feynman diagrams which contribute to the 2PN Lagrangian through the use of power counting in $G$ and $v^{2}$. The calculation involved 21 distinct diagrams, and we encountered integrals which correspond to one-loop and two-loop Feynman integrals. All diagrams could be computed with only two master integrals. This demonstrates the efficiency of the EFT method for the calculation of the 2PN Lagrangian.

Instead of a usual Lorentz covariant metric parametrization, we employed a temporal Kaluza-Klein parametrization of the metric, proposed by Kol and Smolkin [36, 37]. When compared to the $h_{\mu \nu}$ parametrization used in [19], we found that the KS variables reduced the number of diagrams by four at $\mathcal{O}\left(G^{3} v^{0}\right)$ and avoided any propagator insertions in the three-graviton diagrams at $\mathcal{O}\left(G^{2} v^{2}\right)$. This reduced the amount of calculation significantly at $2 \mathrm{PN}$. More generally, we conclude that the KS variables will significantly improve any calculations which are performed at higher order using NRGR methods. This is due to (1) the simple propagators obtained with this gauge and parametrization choice, (2) the suppression of $\phi^{n}$ vertices by one order in the PN expansion due to two time derivatives acting on the vertex, (3) not requiring time insertions in the $\phi^{n}$ topologies at the next order in the PN expansion, and (4) the advantageous compounding effect of the former points when higher order expansions are calculated.

An interesting extension of the current calculation is to compute the $3 \mathrm{PN}$ potential. Analogously to $2 \mathrm{PN}$, where the four-point function formed diagrams which were the most difficult to evaluate, the hardest part of the calculation at $3 \mathrm{PN}$ is expected to involve a gravitational four-point function in $\mathcal{O}\left(G^{4} v^{0}\right)$ diagrams. Also, the number of diagrams one must evaluate at $3 \mathrm{PN}$ is much greater. This means more terms in the action will have to be computed to account for new vertices at $\mathcal{O}\left(G^{4} v^{0}\right), \mathcal{O}\left(G^{3} v^{2}\right), \mathcal{O}\left(G^{2} v^{4}\right)$, and $\mathcal{O}\left(G v^{6}\right)$. Our preliminary estimates show there are more than 100 diagrams at $3 \mathrm{PN}$, compared to the 21 at $2 \mathrm{PN}$. At $3 \mathrm{PN}$, it will again be the case that a reduction in the number of diagrams can be achieved if the KS variables are employed. So while the $3 \mathrm{PN}$ calculation may prove challenging, the relative ease of the calculation at $2 \mathrm{PN}$ indicates that the NRGR calculation of the 3PN potential can be done.

\section{Acknowledgements}

The authors thank Walter Goldberger for many insightful discussions and comments. This work has been supported 
in part by Grant No. DE-FG-02-92ER40704 from the US Department of Energy.

[1] A. Abramovici et al., Science 256, 325 (1992), http://www.ligo.caltech.edu/

[2] A. Giazotto, Nucl. Instrum. Meth. A 289, 518 (1990), http://www.virgo.infn.it/

[3] B. Willke et al., Class. Quant. Grav. 19, 1377 (2002), http://geo600.aei.mpg.de//

[4] M. Ando et al. [TAMA Collaboration], Phys. Rev. Lett. 86, 3950 (2001) arXiv:astro-ph/0105473, http://tamago.mtk.nao.ac.jp/

[5] P. J. Sutton [LIGO Scientific Collaboration], J. Phys. Conf. Ser. 110, 062024 (2008), F. Acernese et al. [Virgo Collaboration], arXiv:0803.0376 [gr-qc], B. Abbott et al. [LIGO Scientific Collaboration], arXiv:0807.2834 [gr-qc], T. Akutsu et al. [TAMA Collaboration], Phys. Rev. D 74, 122002 (2006) arXiv:gr-qc/0610064].

[6] K. Danzmann and A. Rudiger, Class. Quant. Grav. 20, S1 (2003), http://lisa.nasa.gov/

[7] J. R. Gair, L. Barack, T. Creighton, C. Cutler, S. L. Larson, E. S. Phinney and M. Vallisneri, Class. Quant. Grav. 21, S1595 (2004) arXiv:gr-qc/0405137.

[8] T. Creighton, Class. Quant. Grav. 20, S853 (2003), http://www.ligo.caltech.edu/advLIGO/

[9] M. Maggiore, "Gravitational Waves. Vol. 1: Theory and Experiments," Oxford University Press, October $200 \%$.

[10] K. Belczynski, V. Kalogera, F. A. Rasio, R. E. Taam and T. Bulik, arXiv:astro-ph/0612032

[11] F. Pretorius, arXiv:0710.1338 [gr-qc].

[12] B. Brügmann, W. Tichy and N. Jansen, Phys. Rev. Lett. 92, 211101 (2004) arXiv:gr-qc/0312112, F. Pretorius, Phys. Rev. Lett. 95, 121101 (2005) arXiv:gr-qc/0507014, M. Campanelli, C. O. Lousto, P. Marronetti and Y. Zlochower, Phys. Rev. Lett. 96, 111101 (2006) arXiv:gr-qc/0511048, J. G. Baker, J. Centrella, D. I. Choi, M. Koppitz and J. van Meter, Phys. Rev. Lett. 96, 111102 (2006) arXiv:gr-qc/0511103, P. Diener et al., Phys. Rev. Lett. 96, 121101 (2006) arXiv:gr-qc/0512108, M. Campanelli, C. O. Lousto and Y. Zlochower, Phys. Rev. D 73, 061501(R) (2006) arXiv:gr-qc/0601091], J. G. Baker, J. Centrella, D. I. Choi, M. Koppitz and J. van Meter, Phys. Rev. D 73, 104002 (2006) arXiv:gr-qc/0602026.

[13] K. D. Kokkotas and B. G. Schmidt, Living Rev. Rel. 2, 2 (1999) arXiv:gr-qc/9909058.

[14] L. Blanchet, Living Rev. Rel. 5, 3 (2002), Update: Living Rev. Rel. 9, 4 (2006), arXiv:gr-qc/0202016.

[15] T. Futamase and Y. Itoh, Living Rev. Rel. 10, 2 (2007).

[16] C. Cutler et al., Phys. Rev. Lett. 70, 2984 (1993) arXiv:astro-ph/9208005.

[17] E. Poisson, Phys. Rev. D 52, 5719 (1995) [Addendum-ibid. D 55, 7980 (1997)] arXiv:gr-qc/9505030, E. Poisson and C. M. Will, Phys. Rev. D 52, 848 (1995) arXiv:gr-qc/9502040, A. Krolak, K. D. Kokkotas and G. Schäfer, Phys. Rev. D 52, 2089 (1995) arXiv:gr-qc/9503013.

[18] M. Boyle et al., Phys. Rev. D 76, 124038 (2007) arXiv:0710.0158 [gr-qc]].

[19] W. D. Goldberger and I. Z. Rothstein, Phys. Rev. D 73, 104029 (2006) arXiv:hep-th/0409156.

[20] W. D. Goldberger and I. Z. Rothstein, Gen. Rel. Grav. 38, 1537 (2006) [Int. J. Mod. Phys. D 15, 2293 (2006)] arXiv:hep-th/0605238.

[21] W. D. Goldberger, arXiv:hep-ph/0701129.

[22] T. Damour and N. Deruelle, C. R. Acad. Sci. Ser. II 293, 537 (1981), T. Damour, C. R. Acad. Sci. Ser. II 294, 1355 (1982), T. Damour, in "Gravitational Radiation," eds. N. Deruelle and T. Piran, Amsterdam, Netherlands: North-Holland (1983), S. M. Kopeikin, Sov. Astron. 29, 516 (1985), T. Damour and G. Schäfer, Gen. Relativ. Gravit. 17, 879 (1985), L. Blanchet, G. Faye and B. Ponsot, Phys. Rev. D 58, 124002 (1998) arXiv:gr-qc/9804079, Y. Itoh, T. Futamase and H. Asada, Phys. Rev. D 63, 064038 (2001) arXiv:gr-qc/0101114, M. E. Pati and C. M. Will, Phys. Rev. D 65, 104008 (2002) arXiv:gr-qc/0201001.

[23] P. Jaranowski and G. Schäfer, Phys. Rev. D 57, 7274 (1998) [Erratum-ibid. D 63, 029902 (2001)] arXiv:gr-qc/9712075, P. Jaranowski and G. Schäfer, Phys. Rev. D 60, 124003 (1999) arXiv:gr-qc/9906092, T. Damour, P. Jaranowski and G. Schäfer, Phys. Rev. D 62, 021501 (2000) [Erratum-ibid. D 63, 029903 (2001)] arXiv:gr-qc/0003051], T. Damour, P. Jaranowski and G. Schäfer, Phys. Rev. D 63, 044021 (2001) [Erratum-ibid. D 66, 029901 (2002)] arXiv:gr-qc/0010040, T. Damour, P. Jaranowski and G. Schäfer, Phys. Lett. B 513, 147 (2001) arXiv:gr-qc/0105038.

[24] L. Blanchet and G. Faye, Phys. Lett. A 271, 58 (2000) arXiv:gr-qc/0004009], L. Blanchet and G. Faye, Phys. Rev. D 63, 062005 (2001) arXiv:gr-qc/0007051, V. C. de Andrade, L. Blanchet and G. Faye, Class. Quant. Grav. 18, 753 (2001) arXiv:gr-qc/0011063], L. Blanchet, T. Damour and G. Esposito-Farese, Phys. Rev. D 69, 124007 (2004) arXiv:gr-qc/0311052.

[25] Y. Itoh and T. Futamase, Phys. Rev. D 68, 121501(R) (2003) arXiv:gr-qc/0310028, Y. Itoh, Phys. Rev. D 69, 064018 (2004) arXiv:gr-qc/0310029. 
[26] G. Schäfer, Phys. Lett. A 123, 336 (1987).

[27] T. Mitchell and C. M. Will, Phys. Rev. D 75, 124025 (2007) arXiv:0704.2243 [gr-qc]].

[28] L. Blanchet, Phys. Rev. D 54, 1417 (1996) [Erratum-ibid. D 71, 129904 (2005)] arXiv:gr-qc/9603048, L. Blanchet, Class. Quant. Grav. 15, 113 (1998) [Erratum-ibid. 22, 3381 (2005)] arXiv:gr-qc/9710038], L. Blanchet, G. Faye, B. R. Iyer and B. Joguet, Phys. Rev. D 65, 061501 (2002) [Erratum-ibid. D 71, 129902 (2005)] [arXiv:gr-qc/0105099].

[29] R. A. Porto, Phys. Rev. D 73, 104031 (2006) arXiv:gr-qc/0511061, R. A. Porto and I. Z. Rothstein, Phys. Rev. Lett. 97, 021101 (2006) arXiv:gr-qc/0604099], R. A. Porto and I. Z. Rothstein, arXiv:0712.2032 [gr-qc], R. A. Porto and I. Z. Rothstein, arXiv:0802.0720 [gr-qc], R. A. Porto and I. Z. Rothstein, Phys. Rev. D 78, 044013 (2008) arXiv:0804.0260 [gr-qc]].

[30] W. D. Goldberger and I. Z. Rothstein, Phys. Rev. D 73, 104030 (2006) arXiv:hep-th/0511133, R. A. Porto, Phys. Rev. D 77, 064026 (2008) arXiv:0710.5150 [hep-th]].

[31] Y. Z. Chu, W. D. Goldberger and I. Z. Rothstein, JHEP 0603, 013 (2006) arXiv:hep-th/0602016.

[32] V. Cardoso, O. J. C. Dias and P. Figueras, arXiv:0807.2261 [hep-th].

[33] C. R. Galley and B. L. Hu, arXiv:0801.0900 [gr-qc].

[34] U. Cannella and R. Sturani, arXiv:0808.4034 [gr-qc].

[35] H. Sanctuary and R. Sturani, arXiv:0809.3156 [gr-qc].

[36] B. Kol and M. Smolkin, Phys. Rev. D 77, 064033 (2008) arXiv:0712.2822 [hep-th]].

[37] B. Kol and M. Smolkin, Class. Quant. Grav. 25, 145011 (2008) arXiv:0712.4116 [hep-th]].

[38] M. Levi, arXiv:0802.1508 [gr-qc].

[39] B. R. Holstein and J. F. Donoghue, Phys. Rev. Lett. 93, 201602 (2004) arXiv:hep-th/0405239.

[40] M. E. Peskin and D. V. Schroeder, Reading, USA: Addison-Wesley (1995).

[41] V. A. Smirnov, "Feynman integral calculus," Berlin, Germany: Springer (2006).

[42] B. M. Barker and R. F. O'Connell, Phys. Lett. A 78, 231 (1980). 